\title{
Discrete exact solutions to some nonlinear differential-difference equations via the $\left(G^{\prime} / G\right)$-expansion method
}

\author{
İsmail Aslan \\ Department of Mathematics, Izmir Institute of Technology, Urla, Izmir 35430, Turkey
}

\section{A R T I C L E I N F O}

\section{Keywords:}

$\left(\mathrm{G}^{\prime} / \mathrm{G}\right)$-expansion method

Nonlinear differential-difference equation

Discrete nonlinear Schrödinger equation

Toda lattice equation

Lattice equation

\begin{abstract}
A B S T R A C T
We extended the $\left(G^{\prime} / G\right)$-expansion method to two well-known nonlinear differentialdifference equations, the discrete nonlinear Schrödinger equation and the Toda lattice equation, for constructing traveling wave solutions. Discrete soliton and periodic wave solutions with more arbitrary parameters, as well as discrete rational wave solutions, are revealed. It seems that the utilized method can provide highly accurate discrete exact solutions to NDDEs arising in applied mathematical and physical sciences.
\end{abstract}

(c) 2009 Elsevier Inc. All rights reserved.

\section{Introduction}

The study of nonlinear differential-difference equations (NDDEs) has attracted a wide interest since the original work of Fermi et al. in the 1950s [1]. NDDEs, treated as models of some physical phenomena, have become the focus of common concern and played a crucial role in various branches of applied sciences such as biophysics, condensed matter physics and mechanical engineering, and in different physical problems such as atomic chains, currents in electrical networks and molecular crystals [2-5]. We can also encounter NDDEs in numerical simulation of soliton dynamics in high energy physics. Paying more attention to such equations, many integrable NDDEs have been proposed by the researchers, such as the Toda lattice equation [6], the Volterra lattice equation [7], the discrete KdV equation [8] and the Ablowitz-Ladik lattice equation [9]. Contrary to difference equations being fully discrete, NDDEs are semi-discrete with some (or all) of their space variables discrete while time is usually continuous.

The investigation of discrete exact analytic solutions of NDDEs plays an important role in the study of their corresponding nonlinear physical phenomena. Many effective analytic methods for solving nonlinear evolution equations (NEEs) are available in the literature [10-23]. How to extend newly developed methods for NEEs to solve NDDEs is an interesting and important issue. To mention some of the research made on this direction, Hu and Ma [24] used Hirota's bilinear method to construct special soliton-like solutions of the Toeplitz lattice. With the development of computer algebra systems, Baldwin and his co-workers [25] devised an algorithm for discrete nonlinear models in terms of a tanh function. Their work can be thought as a breakthrough for solving NDDEs symbolically. By applying the Jacobi elliptic function expansion method, explicit and exact traveling wave solutions to three NDDEs are found by Liu et al. [26]. An extended Jacobian elliptic function algorithm is presented by Dai et al. [27]. Xie et al. [28] studied the discrete sine-Gordon equation implementing a method based on the Riccati equation expansion. Zhu $[29,30]$ extended the Exp-function method to NDDEs to obtain physically important solutions. Yang et al. [31] considered two nonlinear lattice equations using the so-called ADM-Padé technique. The homotopy perturbation method is applied by Zhu et al. [32] to a nonlinear differential-difference equation arising in nanotechnology. Zhen [33] developed a discrete tanh method. Lately, Aslan [34] presented a generalization of the extended simplest equation method to NDDEs, and so on.

E-mail address: ismailaslan@iyte.edu.tr 
Whilst there has been considerable work done on finding exact solutions to NEEs, little work is being done to symbolically compute exact discrete solutions of NDDEs as far as we could verify. Not long ago, Wang et al. [35] proposed the (G'/G)expansion method to obtain exact solutions of NEEs arising in mathematical physics. Based on Wang et al.'s pioneer work and his followers, the $\left(\mathrm{G}^{\prime} / \mathrm{G}\right)$-expansion method has become popular in the research community, and there has been a number of studies refining the initial idea [36-50]. The method is efficient, reliable and faster by means of a symbolic computation system. It is also powerful for taking full advantage of linear theory of ordinary differential equations.

It is hard to extend an analytic method for NDDEs because of the difficulty which usually arises when we search for iterative relations from indices $n$ to $n \pm i$. Fortunately, Zhang et al. [51] have successfully extended the ( $\left.G^{\prime} / G\right)$-expansion method to the $(2+1)$-dimensional TLE and the discrete Wadati equation. Our objective in this study is to further extend the $\left(\mathrm{G}^{\prime} / \mathrm{G}\right)$ expansion method to two physically important NDDEs for the first time. We slightly adjust the proposed algorithm in [51] by considering a third case for the inclusion of rational solutions as well.

The rest of this paper is organized as follows: in Section 2, we describe the $\left(G^{\prime} / G\right)$-expansion method for finding exact discrete traveling wave solutions of NDDEs, and state the main steps. In Section 3, we illustrate our procedure in detail with the discrete nonlinear Schrödinger equation (DNSE) and the Toda lattice equation (TLE). Finally, some conclusions are given in Section 4.

\section{The $\left(G^{\prime} / G\right)$-expansion method for NDDEs}

Let us consider a system of $M$ polynomial NDDEs in the form

$$
P \mathbf{u}_{n+p_{1}}(\mathbf{x}), \ldots, \mathbf{u}_{n+p_{k}}(\mathbf{x}), \ldots, \mathbf{u}_{n+p_{1}}^{\prime}(\mathbf{x}), \ldots, \mathbf{u}_{n+p_{k}}^{\prime}(\mathbf{x}), \ldots, \mathbf{u}_{n+p_{1}}^{(r)}(\mathbf{x}), \ldots, \mathbf{u}_{n+p_{k}}^{(r)}(\mathbf{x})=0,
$$

where the dependent variable $\mathbf{u}_{\mathbf{n}}$ have $M$ components $u_{i, \mathbf{n}}$ and so do its shifts, the continuous variable $\mathbf{x}$ has $N$ components $x_{i}$, the discrete variable $\mathbf{n}$ has $Q$ components $n_{j}$, the $k$ shift vectors $\mathbf{p}_{i} \in \mathbb{Z}^{Q}$, and $\mathbf{u}^{(r)}(\mathbf{x})$ denotes the collection of mixed derivative terms of order $r$. We summarize our five step-algorithm as follows:

Step 1: For finding traveling wave solutions to Eq. (1), we introduce the wave transformation

$$
\mathbf{u}_{\mathbf{n}+\mathbf{p}_{s}}(\mathbf{x})=\mathbf{U}_{\mathbf{n}+\mathbf{p}_{s}}\left(\xi_{\mathbf{n}}\right), \quad \xi_{\mathbf{n}}=\sum_{i=1}^{Q} d_{i} n_{i}+\sum_{j=1}^{N} c_{j} x_{j}+\zeta, \quad(s=1,2, \ldots, k),
$$

where the coefficients $c_{1}, c_{2}, \ldots, c_{N}, d_{1}, d_{2}, \ldots, d_{Q}$ and the phase $\zeta$ are all constants. Then, Eq. (1) takes the form

$$
P \mathbf{U}_{\mathbf{n}+\mathbf{p}_{1}}\left(\xi_{\mathbf{n}}\right), \ldots, \mathbf{U}_{n+p_{k}}\left(\xi_{\mathbf{n}}\right), \ldots, \mathbf{U}_{\mathbf{n}+\mathbf{p}_{1}}^{\prime}\left(\xi_{\mathbf{n}}\right), \ldots, \mathbf{U}_{\mathbf{n}+\mathbf{p}_{k}}\left(\xi_{\mathbf{n}}\right), \ldots, \mathbf{U}_{\mathbf{n}+\mathbf{p}_{1}}^{(r)}\left(\xi_{\mathbf{n}}\right), \ldots, \mathbf{U}_{\mathbf{n}+\mathbf{p}_{k}}^{(r)}\left(\xi_{\mathbf{n}}\right)=0 .
$$

Step 2: We assume that the solution of Eq. (3) can be expressed in the finite series expansion

$$
\mathbf{U}_{\mathbf{n}}\left(\xi_{\mathbf{n}}\right)=\sum_{l=0}^{m} \boldsymbol{a}_{l}\left(\frac{G^{\prime}\left(\xi_{\mathbf{n}}\right)}{G\left(\xi_{\mathbf{n}}\right)}\right)^{l}, \quad a_{m} \neq 0
$$

where $m$ (a positive integer) and $a_{l}$ 's are constants to be determined later, $G\left(\xi_{\mathbf{n}}\right)$ is the general solution of the auxiliary equation

$$
G^{\prime \prime}\left(\xi_{n}\right)+\lambda G^{\prime}\left(\xi_{n}\right)+\mu G\left(\xi_{n}\right)=0
$$

in which $\lambda$ and $\mu$ are constants to be specified later and prime denotes derivative with respect to $\xi_{\mathbf{n}}$. The general solution of Eq. (5) is well-known for us, and hence we get the following cases:

$$
\begin{aligned}
& \frac{G^{\prime}\left(\xi_{\mathbf{n}}\right)}{G\left(\xi_{\mathbf{n}}\right)}=\frac{\sqrt{\lambda^{2}-4 \mu}}{2}\left(\frac{C_{1} \cosh \left(\frac{\sqrt{\lambda^{2}-4 \mu}}{2} \xi_{\mathbf{n}}\right)+C_{2} \sinh \left(\frac{\sqrt{\lambda^{2}-4 \mu}}{2} \xi_{\mathbf{n}}\right)}{C_{1} \sinh \left(\frac{\sqrt{\lambda^{2}-4 \mu}}{2} \xi_{\mathbf{n}}\right)+C_{2} \cosh \left(\frac{\sqrt{\lambda^{2}-4 \mu}}{2} \xi_{\mathbf{n}}\right)}\right)-\frac{\lambda}{2}, \quad \lambda^{2}-4 \mu>0, \\
& \frac{G^{\prime}\left(\xi_{\mathbf{n}}\right)}{G\left(\xi_{\mathbf{n}}\right)}=\frac{\sqrt{4 \mu-\lambda^{2}}}{2}\left(\frac{-C_{1} \sin \left(\frac{\sqrt{4 \mu-\lambda^{2}}}{2} \xi_{\mathbf{n}}\right)+C_{2} \cos \left(\frac{\sqrt{4 \mu-\lambda^{2}}}{2} \xi_{\mathbf{n}}\right)}{C_{1} \cos \left(\frac{\sqrt{4 \mu-\lambda^{2}}}{2} \xi_{\mathbf{n}}\right)+C_{2} \sin \left(\frac{\sqrt{4 \mu-\lambda^{2}}}{2} \xi_{\mathbf{n}}\right)}\right)-\frac{\lambda}{2}, \quad \lambda^{2}-4 \mu<0, \\
& \frac{G^{\prime}\left(\xi_{\mathbf{n}}\right)}{G\left(\xi_{\mathbf{n}}\right)}=\frac{C_{1}}{C_{1} \xi_{\mathbf{n}}+C_{2}}-\frac{\lambda}{2}, \quad \lambda^{2}-4 \mu=0,
\end{aligned}
$$

where $C_{1}$ and $C_{2}$ are arbitrary constants.

Step 3: A simple calculation leads to the identity

$$
\xi_{\mathbf{n}+\mathbf{p}_{s}}=\xi_{\mathbf{n}}+\varphi_{s}, \quad \varphi_{s}=p_{s 1} d_{1}+p_{s 2} d_{2}+\cdots+p_{s Q} d_{Q},
$$


where $p_{s j}$ is the $j$ th component of the shift vector $\mathbf{p}_{s}$. Thus, using the trigonometric/hyperbolic function identities and considering the functions $(6 a-c)$ as well as (7), we derive uniform formulas

$$
\begin{aligned}
& \mathbf{U}_{\mathbf{n}+\mathbf{p}_{s}}\left(\xi_{\mathbf{n}}\right)=\sum_{l=0}^{m} a_{l}\left(\frac{\frac{\lambda}{2}+\frac{G^{\prime}\left(\xi_{\mathbf{n}}\right)}{G\left(\xi_{\mathbf{n}}\right)}+\frac{\sqrt{\lambda^{2}-4 \mu}}{2} \tanh \left(\frac{\sqrt{\lambda^{2}-4 \mu}}{2} \varphi_{s}\right)}{1+\frac{2}{\sqrt{\lambda^{2}-4 \mu}}\left(\frac{\lambda}{2}+\frac{G^{\prime}\left(\xi_{\mathbf{n}}\right)}{G\left(\xi_{\mathbf{n}}\right)}\right) \tanh \left(\frac{\sqrt{\lambda^{2}-4 \mu}}{2} \varphi_{s}\right)}-\frac{\lambda}{2}\right)^{l}, \quad a_{m} \neq 0, \quad \lambda^{2}-4 \mu>0, \\
& \mathbf{U}_{\mathbf{n}+\mathbf{p}_{s}}\left(\xi_{\mathbf{n}}\right)=\sum_{l=0}^{m} a_{l}\left(\frac{\frac{\lambda}{2}+\frac{G^{\prime}\left(\xi_{\mathbf{n}}\right)}{G\left(\xi_{\mathbf{n}}\right)}-\frac{\sqrt{4 \mu-\lambda^{2}}}{2} \tan \left(\frac{\sqrt{4 \mu-\lambda^{2}}}{2} \varphi_{s}\right)}{1+\frac{2}{\sqrt{4 \mu-\lambda^{2}}}\left(\frac{\lambda}{2}+\frac{G^{\prime}\left(\xi_{\mathbf{n}}\right)}{G\left(\xi_{\mathbf{n}}\right)}\right) \tan \left(\frac{\sqrt{4 \mu-\lambda^{2}}}{2} \varphi_{s}\right)}-\frac{\lambda}{2}\right)^{l}, \quad a_{m} \neq 0, \quad \lambda^{2}-4 \mu<0, \\
& \mathbf{U}_{\mathbf{n}+\mathbf{p}_{s}}\left(\xi_{\mathbf{n}}\right)=\sum_{l=0}^{m} a_{l}\left(\left(\left(\frac{\lambda}{2}+\frac{G^{\prime}\left(\xi_{\mathbf{n}}\right)}{G\left(\xi_{\mathbf{n}}\right)}\right) /\left(1+\left(\frac{\lambda}{2}+\frac{G^{\prime}\left(\xi_{\mathbf{n}}\right)}{G\left(\xi_{\mathbf{n}}\right)}\right) \varphi_{s}\right)\right)-\frac{\lambda}{2}\right)^{l}, \quad a_{m} \neq 0, \quad \lambda^{2}-4 \mu=0 .
\end{aligned}
$$

Step 4: From Eq. (3), we can easily determine the degree $m$ of Eqs. (4) and (8a-c) by balancing the highest order nonlinear $\operatorname{term}(s)$ and the highest-order derivative term in $\mathbf{U}_{\mathbf{n}}\left(\xi_{\mathbf{n}}\right)$ as in the continuous case. The leading terms of $\mathbf{U}_{\mathbf{n}+\mathbf{p}_{s}}\left(\mathbf{p}_{s} \neq 0\right)$ will not effect the balance since $\mathbf{U}_{\mathbf{n}+\mathbf{p}_{s}}$ can be interpreted as being of degree zero in $\left(G^{\prime}\left(\xi_{\mathbf{n}}\right) / G\left(\xi_{\mathbf{n}}\right)\right)$.

Step 5: Substituting the ansatze (4) and (8a-c) along with (5) into Eq. (3), then setting the coefficients of $\left(G^{\prime}\left(\xi_{\mathbf{n}}\right) / G\left(\xi_{\mathbf{n}}\right)\right)^{l}(l=0,1,2, \ldots)$ to zero, we obtain a system of nonlinear algebraic equations from which the undetermined constants $a_{l}, d_{i}, c_{j}, \lambda$ and $\mu$ can be explicitly found. Finally, we substitute these values into expression (4) and find traveling wave solutions to Eq. (1).

\section{Applications}

We illustrate the effectiveness and the advantages of our method by giving two examples.

\subsection{The discrete nonlinear Schrödinger equation}

An important model for discrete solitons is the integrable DNSE [9,52]

$$
i \frac{d u_{n}(t)}{d t}=\left(u_{n+1}(t)-2 u_{n}(t)+u_{n-1}(t)\right)-\left|u_{n}(t)\right|^{2}\left(u_{n+1}(t)+u_{n-1}(t)\right),
$$

where $u_{n}(t)=u(n, t)$ is the displacement of the $n$th particle from the equilibrium position. Eq. (9) was established by using the inverse scattering method [53], and has many interesting rich mathematical properties and physical applications including dynamics of an harmonic lattice [54], self-trapping on a dimer [4], and pulse dynamics in nonlinear optics [55]. The authors [56-59] successfully studied Eq. (9) by using the Exp-function and the Jacobian elliptic function expansion methods.

For solving the DNSE (9), we first make the traveling wave transformation

$$
u_{n}=e^{i \theta_{n}} \phi_{n}\left(\xi_{n}\right), \quad \theta_{n}=d_{1} n+c_{1} t+\zeta_{1}, \quad \xi_{n}=d_{2} n+c_{2} t+\zeta_{2}
$$

and

$$
u_{n+1}=e^{i \theta_{n}} e^{i d_{1}} \phi_{n+1}\left(\xi_{n}\right), \quad u_{n-1}=e^{i \theta_{n}} e^{-i d_{1}} \phi_{n-1}\left(\xi_{n}\right),
$$

where $d_{1}$ and $c_{1}$ are the wave number of the carrier wave and the frequency, $c_{2}$ and $d_{2}$ are related to the group velocity and the pulse width, $\zeta_{1}$ and $\zeta_{2}$ denote the initial phases. Now, using the Euler formula $e^{ \pm i d_{1}}=\cos d_{1} \pm i \sin d_{1}$, Eq. (9) turns into the system

$$
\left\{\begin{array}{l}
c_{1} \phi_{n}+\cos \left(d_{1}\right)\left(1-\phi_{n}^{2}\right)\left(\phi_{n+1}+\phi_{n-1}\right)-2 \phi_{n}=0 \\
c_{2} \phi_{n}^{\prime}-\sin \left(d_{1}\right)\left(1-\phi_{n}^{2}\right)\left(\phi_{n+1}-\phi_{n-1}\right)=0
\end{array}\right.
$$

We expand the solution of (12) in the form of (4). Balancing the linear term of the highest order with the highest nonlinear term in (12) leads to $m=1$. Thus, we consider the ansatz

$$
\phi_{n}\left(\xi_{n}\right)=a_{0}+a_{1}\left(\frac{G^{\prime}\left(\xi_{\mathbf{n}}\right)}{G\left(\xi_{\mathbf{n}}\right)}\right), \quad a_{1} \neq 0
$$

for the traveling wave solutions of (12). Now, a case analysis follows:

Case 1: When $\lambda^{2}-4 \mu>0$, from (8a), we have

$$
\phi_{n \pm 1}\left(\xi_{n}\right)=a_{0}+a_{1}\left(\frac{\frac{\lambda}{2}+\frac{G^{\prime}\left(\xi_{\mathbf{n}}\right)}{G\left(\xi_{\mathbf{n}}\right)} \pm \frac{\sqrt{\lambda^{2}-4 \mu}}{2} \tanh \left(\frac{\sqrt{\lambda^{2}-4 \mu}}{2} d_{2}\right)}{1 \pm \frac{2}{\sqrt{\lambda^{2}-4 \mu}}\left(\frac{\lambda}{2}+\frac{G^{\prime}\left(\xi_{\mathbf{n}}\right)}{G\left(\xi_{\mathbf{n}}\right)}\right) \tanh \left(\frac{\sqrt{\lambda^{2}-4 \mu}}{2} d_{2}\right)}-\frac{\lambda}{2}\right),
$$


where the signs $( \pm)$ or $(\mp)$ are ordered vertically from now on. Substituting (13) and (14) along with (5) into (12), clearing the denominator and setting the coefficients of all powers like $\left(G^{\prime}\left(\xi_{\mathbf{n}}\right) / G\left(\xi_{\mathbf{n}}\right)\right)^{i}(0 \leqslant i \leqslant 4)$ to zero, we derive a system of nonlinear algebraic equations for $a_{0}, a_{1}, c_{1}, c_{2}, d_{1}, d_{2}, \lambda$, and $\mu$. Solving the set of algebraic equations (we omit to display them for brevity) simultaneously, we get the solution set

$$
\left\{\begin{array}{l}
c_{1}=2-2 \cos \left(d_{1}\right) \sec h^{2}\left(\frac{\sqrt{\lambda^{2}-4 \mu}}{2} d_{2}\right), \quad c_{2}=\frac{4 \sin \left(d_{1}\right)}{\sqrt{\lambda^{2}-4 \mu}} \tanh \left(\frac{\sqrt{\lambda^{2}-4 \mu}}{2} d_{2}\right) \\
a_{0}=\mp \frac{\lambda}{\sqrt{\lambda^{2}-4 \mu}} \tanh \left(\frac{\sqrt{\lambda^{2}-4 \mu}}{2} d_{2}\right), \quad a_{1}=\mp \frac{2}{\sqrt{\lambda^{2}-4 \mu}} \tanh \left(\frac{\sqrt{\lambda^{2}-4 \mu}}{2} d_{2}\right)
\end{array}\right\}
$$

where $d_{1}$ and $d_{2}$ are arbitrary constants. Hence, from (6a), (10), (13), (14) and (15), we obtain discrete hyperbolic function traveling wave solutions to Eq. (9) as

$$
\begin{aligned}
u_{n, 1}^{\mp}(t)= & \mp \tanh \left(\frac{\sqrt{\lambda^{2}-4 \mu}}{2} d_{2}\right)\left(\frac{C_{1} \cosh \left(\frac{\sqrt{\lambda^{2}-4 \mu}}{2} \xi_{n}\right)+C_{2} \sinh \left(\frac{\sqrt{\lambda^{2}-4 \mu}}{2} \xi_{n}\right)}{C_{1} \sinh \left(\frac{\sqrt{\lambda^{2}-4 \mu}}{2} \xi_{n}\right)+C_{2} \cosh \left(\frac{\sqrt{\lambda^{2}-4 \mu}}{2} \xi_{n}\right)}\right) \\
& \times \exp \left(i\left(d_{1} n+\left(2-2 \cos \left(d_{1}\right) \sec h^{2}\left(\frac{\sqrt{\lambda^{2}-4 \mu}}{2} d_{2}\right)\right) t+\zeta_{1}\right)\right),
\end{aligned}
$$

where $\xi_{n}=d_{2} n+\frac{4 \sin \left(d_{1}\right)}{\sqrt{\lambda^{2}-4 \mu}} \tanh \left(\frac{\sqrt{\lambda^{2}-4 \mu}}{2} d_{2}\right) t+\zeta_{2}, C_{1}$ and $C_{2}$ are arbitrary constants.

Remark 1. As a particular example, if we take $C_{2} \neq 0, C_{1}^{2}<C_{2}^{2}$ in (16) then we get discrete solitary wave solutions to Eq. (9) as

$$
\begin{aligned}
u_{n, 2}^{\mp}(t)= & \mp \tanh \left(\frac{\sqrt{\lambda^{2}-4 \mu}}{2} d_{2}\right) \tanh \left(\frac{\sqrt{\lambda^{2}-4 \mu}}{2}\left(d_{2} n+\frac{4 \sin \left(d_{1}\right)}{\sqrt{\lambda^{2}-4 \mu}} \tanh \left(\frac{\sqrt{\lambda^{2}-4 \mu}}{2} d_{2}\right) t+\zeta_{2}\right)+\tanh ^{-1}\left(\frac{C_{1}}{C_{2}}\right)\right) \\
& \times \exp \left(i\left(d_{1} n+\left(2-2 \cos \left(d_{1}\right) \sec ^{2}\left(\frac{\sqrt{\lambda^{2}-4 \mu}}{2} d_{2}\right)\right) t+\zeta_{1}\right)\right) .
\end{aligned}
$$

Besides, if we set $d_{2}=2 k / \sqrt{\lambda^{2}-4 \mu}, d_{1}=p, \zeta_{1}=\delta$, and $\zeta_{2}=2\left(\zeta-\tanh ^{-1}\left(C_{1} / C_{2}\right)\right) / \sqrt{\lambda^{2}-4 \mu}$ in (17), then we obtain

$$
u_{n, 3}^{\mp}(t)=\mp \tanh (k) \tanh (k n+2 \sin (p) \tanh (k) t+\zeta) \times \exp \left(i\left(p n+\left(2-2 \cos (p) \sec ^{2}(k)\right) t+\delta\right)\right),
$$

which are the known dark solitary wave solutions found by Dai and Zhang [57] and Dai et al. [58] in which they are expressed as (20) and (34), respectively. However, in our case, we note that (18) is derived from a more general solution (16) in the sense that it contains more arbitrary parameters. Similarly, our solution (16) can be reduced to the solution (45) presented in Huang and Liu [59] by a suitable modification.

Case 2: When $\lambda^{2}-4 \mu<0$, from (8b), we have

$$
\phi_{n \pm 1}\left(\xi_{n}\right)=a_{0}+a_{1}\left(\frac{\frac{\lambda}{2}+\frac{G^{\prime}\left(\xi_{\mathbf{n}}\right)}{G\left(\xi_{\mathbf{n}}\right)} \mp \frac{\sqrt{4 \mu-\lambda^{2}}}{2} \tan \left(\frac{\sqrt{4 \mu-\lambda^{2}}}{2} d_{2}\right)}{1 \pm \frac{2}{\sqrt{4 \mu-\lambda^{2}}}\left(\frac{\lambda}{2}+\frac{G^{\prime}\left(\xi_{\mathbf{n}}\right)}{G\left(\xi_{\mathbf{n}}\right)}\right) \tan \left(\frac{\sqrt{4 \mu-\lambda^{2}}}{2} d_{2}\right)}-\frac{\lambda}{2}\right) .
$$

Substituting (13) and (19) along with (5) into (12), clearing the denominator and setting the coefficients of all powers like $\left(G^{\prime}\left(\xi_{\mathbf{n}}\right) / G\left(\xi_{\mathbf{n}}\right)\right)^{i}(0 \leqslant i \leqslant 4)$ to zero, we derive a system of nonlinear algebraic equations for $a_{0}, a_{1}, c_{1}, c_{2}, d_{1}, d_{2}, \lambda$, and $\mu$. Solving the set of algebraic equations simultaneously, we get the solution set

$$
\left\{\begin{array}{l}
c_{1}=2-2 \cos \left(d_{1}\right) \sec ^{2}\left(\frac{\sqrt{4 \mu-\lambda^{2}}}{2} d_{2}\right), \quad c_{2}=\frac{4 \sin \left(d_{1}\right)}{\sqrt{4 \mu-\lambda^{2}}} \tan \left(\frac{\sqrt{4 \mu-\lambda^{2}}}{2} d_{2}\right) \\
a_{0}=\mp \frac{\lambda}{\sqrt{4 \mu-\lambda^{2}}} \tan \left(\frac{\sqrt{4 \mu-\lambda^{2}}}{2} d_{2}\right), \quad a_{1}=\mp \frac{2}{\sqrt{4 \mu-\lambda^{2}}} \tan \left(\frac{\sqrt{4 \mu-\lambda^{2}}}{2} d_{2}\right)
\end{array}\right\},
$$

where $d_{1}$ and $d_{2}$ are arbitrary constants. Hence, from (6b), (10), (13), (19) and (20), we obtain discrete trigonometric function traveling wave solutions to Eq. (9) as 


$$
\begin{aligned}
u_{n, 4}^{\mp}= & \mp \tan \left(\frac{\sqrt{4 \mu-\lambda^{2}}}{2} d_{2}\right)\left(\frac{C_{2} \cos \left(\frac{\sqrt{4 \mu-\lambda^{2}}}{2} \xi_{n}\right)-C_{1} \sin \left(\frac{\sqrt{4 \mu-\lambda^{2}}}{2} \xi_{n}\right)}{C_{1} \cos \left(\frac{\sqrt{4 \mu-\lambda^{2}}}{2} \xi_{n}\right)+C_{2} \sin \left(\frac{\sqrt{4 \mu-\lambda^{2}}}{2} \xi_{n}\right)}\right) \\
& \times \exp \left(i\left(d_{1} n+\left(2-2 \cos \left(d_{1}\right) \sec ^{2}\left(\frac{\sqrt{4 \mu-\lambda^{2}}}{2} d_{2}\right)\right) t+\zeta_{1}\right)\right),
\end{aligned}
$$

where $\xi_{n}=d_{2} n+\frac{4 \sin \left(d_{1}\right)}{\sqrt{4 \mu-\lambda^{2}}} \tan \left(\frac{\sqrt{4 \mu-\lambda^{2}}}{2} d_{2}\right) t+\zeta_{2}, C_{1}$ and $C_{2}$ are arbitrary constants.

Remark 2. In particular, if we take $C_{2} \neq 0, C_{1}^{2}<C_{2}^{2}$ in (21) then we get discrete periodic wave solutions to Eq. (9) as

$$
\begin{aligned}
u_{n, 5}^{\mp}= & \mp \tan \left(\frac{\sqrt{4 \mu-\lambda^{2}}}{2} d_{2}\right) \cot \left(\frac{\sqrt{4 \mu-\lambda^{2}}}{2}\left(d_{2} n+\frac{4 \sin \left(d_{1}\right)}{\sqrt{4 \mu-\lambda^{2}}} \tan \left(\frac{\sqrt{4 \mu-\lambda^{2}}}{2} d_{2}\right) t+\zeta_{2}\right)+\tan ^{-1}\left(\frac{C_{1}}{C_{2}}\right)\right) \\
& \times \exp \left(i\left(d_{1} n+\left(2-2 \cos \left(d_{1}\right) \sec ^{2}\left(\frac{\sqrt{4 \mu-\lambda^{2}}}{2} d_{2}\right)\right) t+\zeta_{1}\right)\right) .
\end{aligned}
$$

Moreover, if we set $d_{2}=2 k / \sqrt{4 \mu-\lambda^{2}}, d_{1}=p, \zeta_{1}=\delta$, and $\zeta_{2}=2\left(\zeta-\tan ^{-1}\left(C_{1} / C_{2}\right)\right) / \sqrt{4 \mu-\lambda^{2}}$ in (22), then we obtain

$$
u_{n, 6}^{\mp}(t)=\mp \tan (k) \cot (k n+2 \sin (p) \tan (k) t+\zeta) \times \exp \left(i\left(p n+\left(2-2 \cos (p) \sec ^{2}(k)\right) t+\delta\right)\right),
$$

which turns out to be the singular solitonic solutions (45) derived in Dai et al. [58]. Likewise, assigning special values to the parameters in our solution (21), we can get many known solutions, for example, in Dai et al. [57] as well as Huang and Liu [59]. We omit to display them for making the discussion short.

Case 3: When $\lambda^{2}-4 \mu=0$, from (8c), we have

$$
\phi_{n \pm 1}\left(\xi_{n}\right)=a_{0}+a_{1}\left(\left(\left(\frac{\lambda}{2}+\frac{G^{\prime}\left(\xi_{\mathbf{n}}\right)}{G\left(\xi_{\mathbf{n}}\right)}\right) /\left(1 \pm\left(\frac{\lambda}{2}+\frac{G^{\prime}\left(\xi_{\mathbf{n}}\right)}{G\left(\xi_{\mathbf{n}}\right)}\right) d_{2}\right)\right)-\frac{\lambda}{2}\right) .
$$

Substituting (13) and (24) along with (5) into (12), clearing the denominator and setting the coefficients of all powers like $\left(G^{\prime}\left(\xi_{\mathbf{n}}\right) / G\left(\xi_{\mathbf{n}}\right)\right)^{i}(0 \leqslant i \leqslant 4)$ to zero, we derive a system of nonlinear algebraic equations for $a_{0}, a_{1}, c_{1}, c_{2}, d_{1}, d_{2}, \lambda$, and $\mu$. Solving the set of algebraic equations simultaneously, we get the solution set

$$
\left\{c_{1}=2\left(1-\cos \left(d_{1}\right)\right), \quad c_{2}=2 d_{2} \sin \left(d_{1}\right), a_{0}=\mp \frac{\lambda d_{2}}{2}, a_{1}=\mp d_{2}\right\},
$$

where $d_{1}$ and $d_{2}$ are arbitrary constants. Hence, from (6c), (10), (13), (24) and (25), we obtain discrete rational wave solutions to Eq. (9) as

$$
u_{n, 7}^{\mp}(t)=\mp \frac{C_{1} d_{2}}{C_{1}\left(d_{2} n+2 d_{2} \sin \left(d_{1}\right) t+\zeta_{2}\right)+C_{2}} \times \exp \left(i\left(d_{1} n+2\left(1-\cos \left(d_{1}\right)\right) t+\zeta_{1}\right)\right),
$$

where $C_{1}$ and $C_{2}$ are arbitrary constants.

Remark 3. We observe that the rational solutions (26) are presented here for the first time and they do not appear in [5759].

\subsection{The Toda lattice equation}

Next, we consider the so-called Toda lattice equation $[6,10,60]$ which reads

$$
\frac{d^{2} u_{n}(t)}{d t^{2}}=\alpha\left(2 e^{-\beta u_{n}(t)}-e^{-\beta u_{n+1}(t)}-e^{-\beta u_{n-1}(t)}\right) .
$$

Since it is difficult to apply the method directly to Eq. (27), we introduce the transformation

$$
e^{-\beta u_{n}(t)}=1+\frac{v_{n}(t)}{\alpha},
$$


which immediately reduces Eq. (27) into

$$
\frac{d^{2}}{d t^{2}} \ln \left(1+\frac{v_{n}(t)}{\alpha}\right)=\beta\left(v_{n+1}(t)-2 v_{n}(t)+v_{n-1}(t)\right) .
$$

Assuming $v_{n}(t)=V_{n}\left(\xi_{n}\right), \xi_{n}=d_{1} n+c_{1} t+\zeta$ in Eq. (29), we get

$$
c_{1}^{2} \frac{d^{2}}{d \xi_{n}^{2}} \operatorname{In}\left(1+\frac{V_{n}\left(\xi_{n}\right)}{\alpha}\right)=\beta\left(V_{n+1}\left(\xi_{n}\right)-2 V_{n}\left(\xi_{n}\right)+V_{n-1}\left(\xi_{n}\right)\right) .
$$

Since the procedure is the same as in the previous section, we will omit most of the details here. Now, by the homogeneous balance principle, we can assume the ansatz

$$
V_{n}\left(\xi_{\mathbf{n}}\right)=a_{0}+a_{1}\left(\frac{G^{\prime}\left(\xi_{\mathbf{n}}\right)}{G\left(\xi_{\mathbf{n}}\right)}\right)+a_{2}\left(\frac{G^{\prime}\left(\xi_{\mathbf{n}}\right)}{G\left(\xi_{\mathbf{n}}\right)}\right)^{2}, \quad a_{2} \neq 0
$$

for the traveling wave solutions of Eq. (30). After a careful analysis, we end up with the following cases:

Case 1: $\lambda^{2}-4 \mu>0$.

Substituting (31) along with $V_{n+1}\left(\xi_{n}\right)$ and $V_{n-1}\left(\xi_{n}\right)$ derived from (8a) into Eq. (30), clearing the denominator and equating the coefficients of $\left(G^{\prime}\left(\xi_{\mathbf{n}}\right) / G\left(\xi_{\mathbf{n}}\right)\right)^{i}(0 \leqslant i \leqslant 10)$ to zero, and solving the resulting algebraic system for $a_{0}, a_{1}, a_{2}, c_{1}, d_{1}, \lambda$, and $\mu$, we get a solution set (denoted in curly brackets from now on) and the corresponding discrete hyperbolic function traveling wave solution to Eq. (27) as follows:

$$
\begin{aligned}
& \left\{a_{0}=\frac{-\left(\alpha \beta+c_{1}^{2} \mu\right)}{\beta}+\frac{c_{1}^{2}\left(\lambda^{2}-4 \mu\right)}{4 \beta} \operatorname{csch}^{2}\left(\frac{\sqrt{\lambda^{2}-4 \mu}}{2} d_{1}\right), \quad a_{1}=-\frac{\lambda c_{1}^{2}}{\beta}, \quad a_{2}=-\frac{c_{1}^{2}}{\beta}\right\},
\end{aligned}
$$

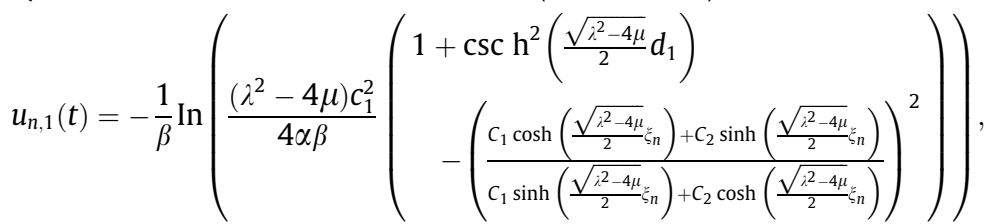

where $\xi_{n}=d_{1} n+c_{1} t+\zeta, C_{1}$ and $C_{2}$ are arbitrary constants.

Remark 4. For instance, if we take $C_{2} \neq 0, C_{1}^{2}<C_{2}^{2}$ in (33) then we get a discrete solitary wave solution to Eq. (27) as

$$
u_{n, 2}(t)=-\frac{1}{\beta} \operatorname{In}\left(\frac{\left(\lambda^{2}-4 \mu\right) c_{1}^{2}}{4 \alpha \beta}\left(1+\operatorname{csch}^{2}\left(\frac{\sqrt{\lambda^{2}-4 \mu}}{2} d_{1}\right)-\tanh ^{2}\left(\frac{\sqrt{\lambda^{2}-4 \mu}}{2}\left(d_{1} n+c_{1} t+\zeta\right)+\tanh ^{-1}\left(\frac{C_{1}}{C_{2}}\right)\right)\right)\right) .
$$

It is clear that our result (34) includes Liu et al.'s [26] result (41) as a special case.

Case 2: $\lambda^{2}-4 \mu<0$.

Substituting (31) along with $V_{n+1}\left(\xi_{n}\right)$ and $V_{n-1}\left(\xi_{n}\right)$ derived from (8b) into Eq. (30), clearing the denominator and equating the coefficients of $\left(G^{\prime}\left(\xi_{\mathbf{n}}\right) / G\left(\xi_{\mathbf{n}}\right)\right)^{i}(0 \leqslant i \leqslant 10)$ to zero, and solving the resulting algebraic system for $a_{0}, a_{1}, a_{2}, c_{1}, d_{1}, \lambda$, and $\mu$, we get a solution set and the corresponding discrete trigonometric function traveling wave solution to Eq. (27) as follows:

$$
\begin{aligned}
& \left\{a_{0}=\frac{-\left(\alpha \beta+c_{1}^{2} \mu\right)}{\beta}-\frac{c_{1}^{2}\left(\lambda^{2}-4 \mu\right)}{4 \beta} \csc ^{2}\left(\frac{\sqrt{4 \mu-\lambda^{2}}}{2} d_{1}\right), \quad a_{1}=-\frac{\lambda c_{1}^{2}}{\beta}, \quad a_{2}=-\frac{c_{1}^{2}}{\beta}\right\}, \\
& u_{n, 3}(t)=-\frac{1}{\beta} \ln \left(\frac{\left(\lambda^{2}-4 \mu\right) c_{1}^{2}}{4 \alpha \beta}\left(\begin{array}{c}
1-\csc ^{2}\left(\frac{\sqrt{4 \mu-\lambda^{2}}}{2} d_{1}\right) \\
+\left(\frac{-c_{1} \sin \left(\frac{\sqrt{4 \mu-\lambda^{2}}}{2} \xi_{n}\right)+C_{2} \cos \left(\frac{\sqrt{4 \mu-\lambda^{2}}}{2} \xi_{n}\right)}{c_{1} \cos \left(\frac{\sqrt{4 \mu-\lambda^{2}}}{2} \xi_{n}\right)+C_{2} \sin \left(\frac{\sqrt{4 \mu-\lambda^{2}}}{2} \xi_{n}\right)}\right)
\end{array}\right)\right),
\end{aligned}
$$

where $\xi_{n}=d_{1} n+c_{1} t+\zeta, C_{1}$ and $C_{2}$ are arbitrary constants. 
Remark 5. In particular, if we take $C_{2} \neq 0, C_{1}^{2}<C_{2}^{2}$ in (36) then we obtain a discrete periodic wave solution to Eq. (27) as

$$
u_{n, 4}(t)=-\frac{1}{\beta} \operatorname{In}\left(\frac{\left(\lambda^{2}-4 \mu\right) c_{1}^{2}}{4 \alpha \beta}\left(1-\csc ^{2}\left(\frac{\sqrt{4 \mu-\lambda^{2}}}{2} d_{1}\right)+\cot ^{2}\left(\frac{\sqrt{4 \mu-\lambda^{2}}}{2}\left(d_{1} n+c_{1} t+\zeta\right)+\tan ^{-1}\left(\frac{C_{1}}{C_{2}}\right)\right)\right)\right) .
$$

We also note that many solutions obtained in Liu et al.'s [26] work can be easily recovered from our solutions (36) and (37) with appropriate choices of the parameters.

Case 3: $\lambda^{2}-4 \mu=0$.

Substituting (31) along with $V_{n+1}\left(\xi_{n}\right)$ and $V_{n-1}\left(\xi_{n}\right)$ derived from (8c) into Eq. (30), clearing the denominator and equating the coefficients of $\left(G^{\prime}\left(\xi_{\mathbf{n}}\right) / G\left(\xi_{\mathbf{n}}\right)\right)^{i}(0 \leqslant i \leqslant 10)$ to zero, and solving the resulting algebraic system for $a_{0}, a_{1}, a_{2}, c_{1}, d_{1}$, and $\lambda$, we get the solution sets and the corresponding discrete rational function traveling wave solutions to Eq. (27) as follows:

$$
\begin{aligned}
& \left\{a_{0}=-\alpha+\frac{1}{4} a_{2}\left(\lambda^{2}-\frac{4}{d_{1}^{2}}\right), \quad a_{1}=\lambda a_{2}, \quad c_{1}=\mp i \sqrt{\beta a_{2}}\right\}, \\
& u_{n, 5}^{\mp}(t)=-\frac{1}{\beta} \operatorname{In}\left(\frac{a_{2}}{\alpha}\left(\frac{C_{1}^{2}}{\left(C_{1}\left(d_{1} n \mp i \sqrt{\beta a_{2}} t+\zeta\right)+C_{2}\right)^{2}}-\frac{1}{d_{1}^{2}}\right)\right),
\end{aligned}
$$

where $C_{1}$ and $C_{2}$ are arbitrary constants.

Remark 6. We also observe that the rational solutions (39) are presented here for the first time and they do not appear in Liu et al. [26].

\section{Conclusion}

Using the $\left(G^{\prime} / G\right)$-expansion method, we have successfully derived various families of discrete exact solutions to the DNSE and the TLE. Some of these solutions are found for the first time. The obtained results with more free parameters include most of the solutions in the open literature as special cases. As a result, the power of the employed method is confirmed. We assured the correctness of the obtained solutions by putting them back into the original equation with the aid of MATHEMATICA, it provides an extra measure of confidence in the results. We predict that the $\left(\mathrm{G}^{\prime} / \mathrm{G}\right)$-expansion method will be a promising method for investigating exact analytic solutions to NDDEs.

\section{References}

[1] E. Fermi, J. Pasta, S. Ulam, Collected Papers of Enrico Fermi II, University of Chicago Press, Chicago, 1965.

[2] A.C. Scott, L. Macheil, Binding energy versus nonlinearity for a small stationary soliton, Phys. Lett. A 98 (1983) 87-88.

[3] W.P. Su, J.R. Schrieffer, A.J. Heege, Solitons in polyacetylene, Phys. Rev. Lett. 42 (1979) 1698-1701

[4] A.S. Davydov, The theory of contraction of proteins under their excitation, J. Theor. Biol. 38 (1973) 559-569.

[5] P. Marquii, J.M. Bilbault, M. Rernoissnet, Observation of nonlinear localized modes in an electrical lattice, Phys. Rev. E 51 (1995) $6127-6133$.

[6] M. Toda, Theory of Nonlinear Lattices, Springer, Berlin, 1989.

[7] M. Wadati, Transformation theories for nonlinear discrete systems, Prog. Suppl. Theor. Phys. 59 (1976) 36-63.

[8] Y. Ohta, R. Hirota, A discrete KdV equation and its casorati determinant solution, J. Phys. Soc. Jpn. 60 (1991) 2095.

[9] M.J. Ablowitz, J. Ladik, Nonlinear differential-difference equation, J. Math. Phys. 16 (1975) 598-603.

[10] M.J. Ablowitz, P.A. Clarkson, Soliton, Nonlinear Evolution Equations and Inverse Scattering, Cambridge University Press, New York, 1991.

[11] R. Hirota, The Direct Method in Soliton Theory, Cambridge University Press, Cambridge, 2004.

[12] W. Malfliet, W. Hereman, The tanh method I: exact solutions of nonlinear evolution and wave equations, Phys. Scripta 54 (1996) 563-568.

[13] G. Adomian, Solving Frontier Problems of Physics: The Decomposition Method, Kluwer, Boston, 1994.

[14] M.A. Abdou, The extended F-expansion method and its application for a class of nonlinear evolution equations, Chaos Soliton Fract. 31 (2007) 95-104.

[15] M.L. Wang, Exact solutions for a compound KdV-Burgers equation, Phys. Lett. A 213 (1996) 279-287.

[16] G.T. Liu, T.Y. Fan, New applications of developed Jacobi elliptic function expansion methods, Phys. Lett. A 345 (2005) 161-166.

[17] J.H. He, X.H. Wu, Exp-function method for nonlinear wave equations, Chaos Soliton Fract. 30 (2006) 700-708.

[18] T. Özis, İ. Aslan, Exact and explicit solutions to the (3+1)-dimensional Jimbo-Miwa equation via the Exp-function method, Phys. Lett. A 372 (2008) 7011-7015.

[19] J.H. He, An approximate solution technique depending on an artificial parameter: a special example, Commun. Nonlinear Sci. Numer. Simul. 3 (1998) 92-97.

[20] İ. Aslan, Generalized solitary and periodic wave solutions to a $(2+1)$-dimensional Zakharov-Kuznetsov equation, Appl. Math. Comput. (2009), doi:10.1016/j.amc.2009.05.037.

[21] A. Biswas, 1-Soliton solution of the $K(m, n)$ equation with generalized evolution, Phys. Lett. A 372 (2008) 4601-4602.

[22] A. Biswas, 1-Soliton solution of the $B(m, n)$ equation with generalized evolution, Commun. Nonlinear Sci. Numer. Simul. 14 (2009) 3226-3229.

[23] A. Biswas, Solitary waves for power-law regularized long-wave equation and $R(m, n)$ equation, Nonlinear Dyn. (2009), doi:10.1007/s11071-009-95482.

[24] X.B. Hu, W.X. Ma, Application of Hirota's bilinear formalism to the Toeplitz lattice-some special soliton-like solutions, Phys. Lett. A 293 (2002) 161165.

[25] D. Baldwin, U. Goktas, W. Hereman, Symbolic computation of hyperbolic tangent solutions for nonlinear differential-difference equations, Comput. Phys. Commun. 162 (2004) 203-217. 
[26] S.K. Liu, Z.T. Fu, Z.G. Wang, S.D. Liu, Periodic solutions for a class of nonlinear differential-difference equations, Commun. Theor. Phys. 49 (2008) 11551158.

[27] C.Q. Dai, J.P. Meng, J.F. Zhang, Symbolic computation of extended Jacobian elliptic function algorithm for nonlinear differential-different equations, Commun. Theor. Phys. 43 (2005) 471-478.

[28] F. Xie, M. Jia, H. Zhao, Some solutions of discrete sine-Gordon equation, Chaos Soliton Fract. 33 (2007) 1791-1795

[29] S.D. Zhu, Exp-function method for the hybrid-lattice system, Int. J. Nonlinear Sci. 8 (2007) 461.

[30] S.D. Zhu, Exp-function method for the discrete mKdV lattice, Int. J. Nonlinear Sci. 8 (2007) 465.

[31] P. Yang, Y. Chen, Z.B. Li, ADM-Padé technique for the nonlinear lattice equations, Appl. Math. Comput. 210 (2009) $362-375$.

[32] S.D. Zhu, Y.M. Chu, S.L. Qiu, The homotopy perturbation method for discontinued problems arising in nanotechnology, Comput. Math. Appl. (2009), doi:10.1016/j.camwa.2009.03.048.

[33] W. Zhen, Discrete tanh method for nonlinear difference-differential equations, Comput. Phys. Commun. 180 (2009) $1104-1108$.

[34] İ. Aslan, A discrete generalization of the extended simplest equation method, Commun. Nonlinear Sci. Numer. Simul. (2009), doi:10.1016/ j.cnsns.2009.08.008.

[35] M. Wang, X. Li, J. Zhang, The ( $\left.\mathrm{G}^{\prime} / \mathrm{G}\right)$-expansion method and traveling wave solutions of nonlinear evolution equations in mathematical physics, Phys. Lett. A 372 (2008) 417-423.

[36] M. Wang, J. Zhang, X. Li, Application of the ( $\left.\mathrm{G}^{\prime} / \mathrm{G}\right)$-expansion to travelling wave solutions of the Broer-Kaup and the approximate long water wave equations, Appl. Math. Comput. 206 (2008) 321-326.

[37] L. Ling-Xiao, W. Ming-Liang, The $\left(G^{\prime} / G\right)$-expansion method and travelling wave solutions for a higher-order nonlinear Schrödinger equation, Appl. Math. Comput. 208 (2009) 440-445

[38] M.S. Bruzon, M.L. Gandarias, New exact solutions for a Benjamin-Bona-Mahony equation, Math. Comput. Sci. Eng. (2008) 189-194.

[39] İ. Aslan, T. Öziş, Analytic study on two nonlinear evolution equations by using the (G'/G)-expansion method, Appl. Math. Comput. 209 (2009) 425-429.

[40] I. Aslan, T. Öziş, On the validity and reliability of the $\left(\mathrm{G}^{\prime} / \mathrm{G}\right)$-expansion method by using higher-order nonlinear equations, Appl. Math. Comput. 211 (2009) 531-536.

[41] T. Öziş, İ. Aslan, Symbolic computation and construction of new exact traveling wave solutions to Fitzhugh-Nagumo and Klein-Gordon equations, Z. Naturforsch. 64a (2009) 15-20.

[42] T. Öziş, İ. Aslan, Symbolic computations and exact and explicit solutions of some nonlinear evolution equations in mathematical physics, Commun. Theor. Phys. 51 (2009) 577-580.

[43] J. Zhang, X. Wei, Y. Lu, A generalized ( $\left.\mathrm{G}^{\prime} / G\right)$-expansion method and its applications, Phys. Lett. A 372 (2008) $3653-3658$.

[44] S. Zhang, W. Wang, J. Tong, A generalized $\left(\mathrm{G}^{\prime} / \mathrm{G}\right)$-expansion method and its application to the $(2+1)$-dimensional Broer-Kaup equations, Appl. Math. Comput. 209 (2009) 399-404.

[45] S. Zhang, J.L. Tong, W. Wang, A generalized ( $\left.G^{\prime} / G\right)$-expansion method for the mKdV equation with variable coefficients, Phys. Lett. A 372 (2008) 22542257.

[46] Z. Yu-Bin, L. Chao, Application of modified ( $\left.G^{\prime} / G\right)$-expansion method to traveling wave solutions for Whitham Broer Kaup-Like equations, Commun. Theor. Phys. 51 (2009) 664-670.

[47] H. Zhang, New application of the $\left(\mathrm{G}^{\prime} / \mathrm{G}\right)$-expansion method, Commun. Nonlinear Sci. Numer. Simul. 14 (2009) $3220-3225$.

[48] W.A. Li, H. Cehn, G.C. Zhang, The $(\omega / g)$-expansion method and its application to Vakhnenko equation, Chin. Phys. B 18 (2009) $400-404$.

[49] X. Liu, L. Tian, Y. Wu, Application of the $\left(\mathrm{G}^{\prime} / \mathrm{G}\right)$-expansion method to two nonlinear evolution equations, Appl. Math. Comput. doi:10.1016/ j.amc.2009.05.019.

[50] I. Aslan, Exact and explicit solutions to some nonlinear evolution equations by utilizing the ( $\left.\mathrm{G}^{\prime} / \mathrm{G}\right)$-expansion method, Appl. Math. Comput. 215 (2009) 857-863.

[51] S. Zhang, L. Dong, J. Ba, Y. Sun, The (G'/G)-expansion method for nonlinear differential-difference equations, Phys. Lett. A 373 (2009) $905-910$.

[52] M.J. Ablowitz, J.F. Ladik, Nonlinear differential-difference equations and Fourier analysis, J. Math. Phys. 17 (1976) $1011-1018$.

[53] M.J. Ablowitz, J.F. Ladik, A nonlinear difference scheme and inverse scattering, Stud. Appl. Math. 55 (1976) $213-229$.

[54] S. Takeno, G. Hori, Self-localized modes in a pure one-dimensional lattice with cubic and quartic lattice anharmonicity, J. Phys. Soc. Jpn. 60 (1991) $947-$ 959.

[55] A. Aceves, C. Angelis, G. Luther, G. Rubenchik, S. Turitsyn, Energy localization in nonlinear fiber arrays: collapse-effect compressor, Phys. Rev. Lett. 75 (1995) 73-76.

[56] C.Q. Dai, Y.Y. Wang, Exact travelling wave solutions of the discrete nonlinear Schrödinger equation and the hybrid lattice equation obtained via the exp-function method, Phys. Scripta 78 (2008) 015013.

[57] C.Q. Dai, J.F. Zhang, Jacobian elliptic function method for nonlinear differential-difference equations, Chaos Soliton Fract. 27 (2006) $1042-1047$.

[58] C.Q. Dai, J.P. Meng, J.F. Zhang, Symbolic computation of extended Jacobian elliptic function algorithm for nonlinear differential-different equations, Commun. Theor. Phys. 43 (2005) 471-478.

[59] W. Huang, Y. Liu, Doubly periodic wave solutions and soliton solutions of Ablowitz-Ladik lattice system, Int. J. Theor. Phys. 47 (2008) 338-349.

[60] G.A. Maugin, Nonlinear Waves in Elastic Crystals, Oxford University Press, New York, 1999. 